\title{
Quantitative Analysis of Point Defects in an Ideal Quasicrystal by Aberration- Corrected Z-contrast STEM
}

\author{
E. Abe*, T. Seki* and S. J Pennycook**
}

*Department of Materials Science \& Engineering, The University of Tokyo, 113-8656, Japan

**Materials Science \& Technology Division, Oak Ridge National Laboratory, Oak Ridge, TN 37830, USA

Quasicrystals are aperiodic long-range ordered solids, whose structure can be reasonably explained according to a generalized form of crystallography by extending its framework into $n$-dimensions $-\mathrm{a}$ hyperspace crystallography with $n>3$ deals a quasicrystal as a periodic structure embedded in a hyperspace. Within this context, a unique elastic degree of freedom arises along the extra dimensions, termed a phason, which is expected to cause atomistic fluctuations that are realized as localized disorder at particular atomic sites in the quasicrystal structure. Quantitative evaluation of these phason-related point defects is one of the important issues to understand thermodynamic stability of quasicrystals, but it is difficult to see the defect configurations in a real-space structure. Here we use aberration-corrected scanning transmission electron microscopy (STEM) to identify the compositions of individual atomic sites (columns) in decagonal $\mathrm{Al}_{72} \mathrm{Ni}_{20} \mathrm{Co}_{8}$, one of the best quasiperiodically ordered materials available today [1]. We indeed find that significant point defects occur even for the highly-ordered quasicrystal, the localized distribution of which is well represented by the decagonal cluster located on the quasilattice with a length-scale of 2nm (Fig. 1, left). Local disorder features appear to be slightly different between the clusters (Fig. 1, right); though, an average structure of the cluster turns out to posses a definite mirror symmetry that governs the occurrence of the disorder sites (Fig. 2). We are able to explain these remarkable disorder features as due to a phasonic perturbation of an energetically stabilized quasicrystal structure.

[1] E. Abe, Y. Yan and S. J. Pennycook, Nature Materials, 3 (2004) 759-767

[2] This work was partly supported by a Grant-in-Aid for Scientific Research of Priority Areas 'Atomic Scale Modification' from the Ministry of Education, Science, Sports and Culture of Japan, and by the Office of Science, Division of Materials Sciences and Engineering, US Department of Energy. 


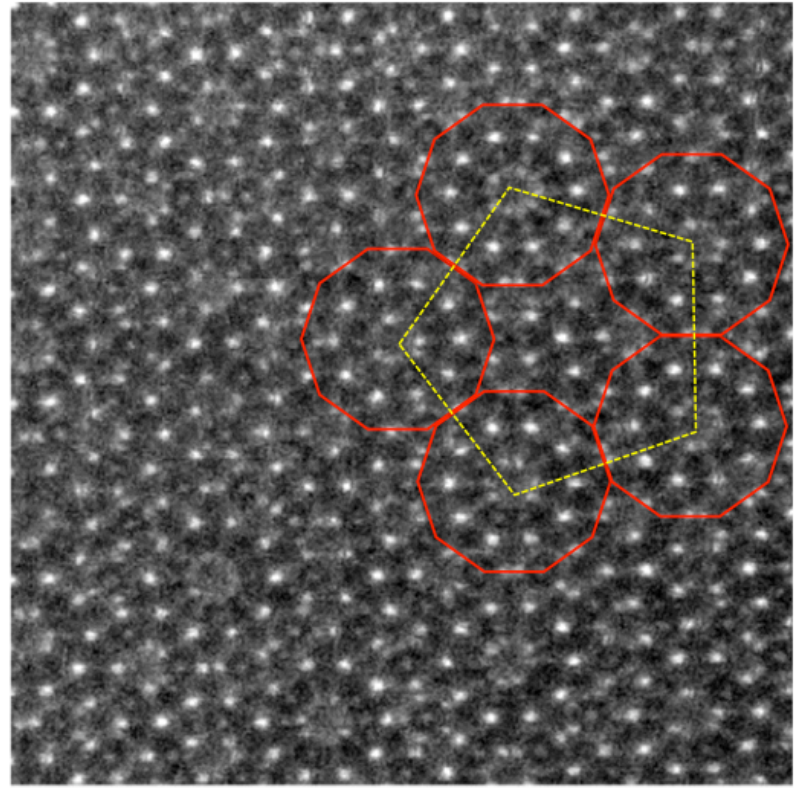

$2 \mathrm{~nm}$

Fig. 1. Left: Z-contrast image of the $\mathrm{Al}_{72} \mathrm{Ni}_{20} \mathrm{Co}_{8}$ decagonal quasicrystal, taken by the Cs-corrected 300kV-STEM (VG-HB603U with Nion corrector). Right : Phsaon-related disorders localized around the center of the $2 \mathrm{~nm}$ clusters.
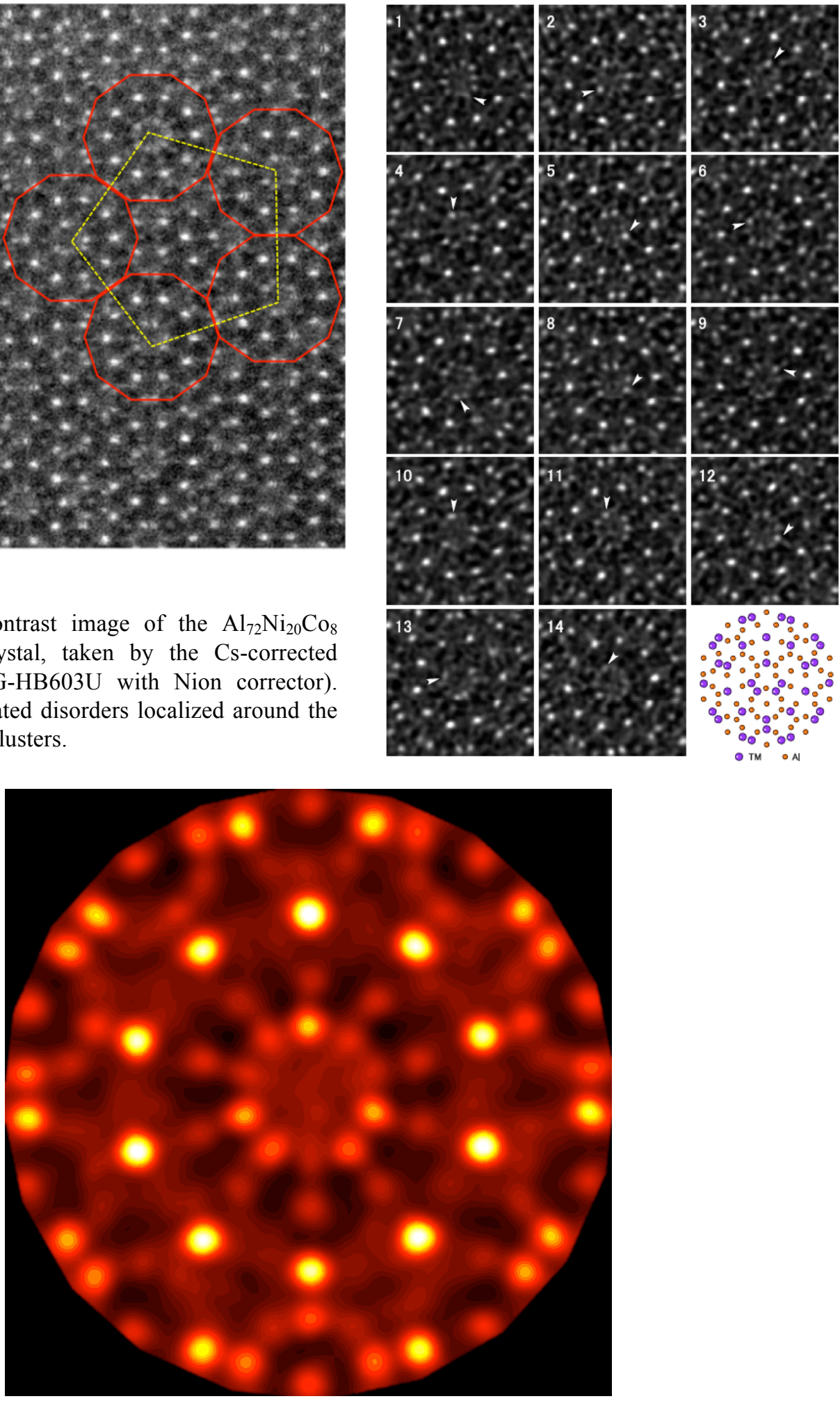

Fig. 1. Average intensity map of the decagonal cluster in the ideal $\mathrm{Al}_{72} \mathrm{Ni}_{20} \mathrm{Co}_{8}$ quasicrystal, showing clearly that the composition fluctuations at individual atomic sites are in accordance with mirror symmetry. Slight deviations from this average symmetry observed between the clusters (Fig. 1, right) can be reasonably interpreted as a consequence of weak phasonic perturbation of an energetically stabilized quasicrystal. 